\title{
Restoration of Multitemporal Short-Exposure Astronomical Images
}

\author{
Michal Haindl ${ }^{1}$ and Stanislava Šimberová ${ }^{2}$ \\ 1 Institute of Information Theory and Automation, \\ Academy of Sciences CR, \\ Prague, CZ182 08, Czech Republic \\ haindl@utia.cas.cz \\ http://www.utia.cas.cz/RO/ \\ 2 Astronomical Institute \\ Academy of Sciences CR, \\ Ondřejov, CZ251 65, Czech Republic \\ ssimbero@sunkl.asu.cas.cz \\ http://www.asu.cas.cz/
}

\begin{abstract}
A multitemporal fast adaptive recursive restoration method based on the underlying spatial probabilistic image model is presented. The method assumes linear degradation model with the unknown possibly non-homogeneous point-spread function and additive noise. Pixels in the vicinity of image steep discontinuities are left unrestored to minimize restoration blurring effect. The method is applied for astronomical sunspot image restoration, where for every ideal undegraded unobservable image several degraded observed images are available.
\end{abstract}

\section{Introduction}

The major degradation of a ground-based telescope is caused by random fluctuations of the optical way between the object space and the image formation device. There are limitations originating mostly in the Earth's atmosphere and also in the instrumentation itself (telescope \& imaging system). Very serious limitation of solar observations is seeing, which has its origin in the Earth's atmosphere. The image quality in the image space decreases by variations of the index of refraction, dominated by the thermal turbulence. Influence of the turbulence in image formation were analyzed since the 1950s, see e.g.[1],[2],[3].

Image degradation by seeing is a very complicated process. The three different aspects can be identified:

1. blurring - image looses its sharpness; it represents the de focusing effect,

2. motion - if the image remains sharp but it is rapidly shifted back and forth,

3. distorsion - substantial parts of the image remain sharp but are shifted relative to each other.

Exposure of $10^{-2} \mathrm{~s}$ and faster can therefore substantially reduce these aspects of solar seeing. Image degradation is described by the changing complex 
point spread function (PSF). PSF of the telescope embodies all the important behaviour of the optical image formation system.

The optical transfer function (OTF) $H=\mathcal{F}(P S F)$ is generally a complex quantity, and in most cases only its modulus $\operatorname{MTF}(u, v)=\|\operatorname{OTF}(u, v)\|$ (modulation transfer function) can be measured. The system transfer function is possible to separate into components originating from diffraction $O T F_{\text {diff }}$, system aberrations $O T F_{a b}$, and from seeing $O T F_{\text {see }}$, then $O T F_{\text {total }}=O T F_{\text {diff }} \times$ $O T F_{a b} \times O T F_{s e e}$.

From the restoration point of view we look on the degradation in general, i.e. there is the only one degradation complex uknown function involving all aspects of degradation. The degradation function model has to differentiate between different types of astronomical observations. There are obviously distinct assumptions in modeling of degradation by the long-exposure stellar objects (exposure time about minutes) and the short-exposure solar images (exposure time about milliseconds).

In our case we focus on modeling of degradation of the short-exposure solar images resulting in recovering an undegraded image. In ground-based solar observations it is often possible to obtain several images of the object under investigation that differ just by the component of PSF originating from seeing. For the reconstruction we suppose a short time sequence of images. The exposure time of the each image is $<15 \mathrm{~ms}$, so we are allowed to involve them into the "short-exposure" ones and suppose a still scene in the image. Our approach to the image restoration is different to direct and indirect techniques like various types of filtering, power spectral equalization, constrained least-squares restoration, maximum entropy restoration, etc. The image restoration task is to recover an unobservable image given the whole sequence differently corrupted images with respect to some statistical criterion.

Many different image restoration methods have been published and several sophisticated algorithms in the last 10 years have been applied. Most of these methods are general purpose image restoration algorithms which cannot benefit from the specific multitemporal solar observation measurement setup. The simplest restoration method is to smooth the data with an isotropic linear or non-linear shift-invariant low-pass filter. Usual filtering techniques (e.g. median filter, Gaussian low pass filter, band pass filters, etc.) tend to blur the location of boundaries. Several methods [4] try to avoid this problem by using a large number of low-pass filters and combining their outputs. Similarly anisotropic diffusion [5],[6] addresses this problem but it is computationally extremely demanding. Image intensity in this method is allowed to diffuse over time, with the amount of diffusion at a point being inversely proportional to the magnitude of local intensity gradient. A nonlinear filtering method developed by Nitzberg and Shiota [7] uses an offset term to displace kernel centers away from presumed edges and thus to preserve them, however it is not easy to propose all filter parameters to perform satisfactory on variety of different images and the algorithm is very slow. In the exceptional case, when the degradation point-spread function is known, the Wiener filter [8] or deconvolution methods [9] can be used. Model- 
based methods use most often Markov random field type of models either in the form of wide sense Markov (regressive models) or strong Markov models. The noncausal regressive model used in [10] has the main problem in time consuming iterative solution based on the conjugate gradient method. Similarly Markov random field based restoration methods [11], [12], [13] require time consuming application of Markov chain Monte Carlo methods. Besides this both approaches have solve the problem when to stop these iterative processes. A similar combination of causal and non-causal regressive models as in this paper was used in [14]. However they assume the homogeneous point-spread function and they identify all parameters simultaneously using extremely time consuming iterations of the EM (expectation maximization) algorithm which is not guaranteed to reach the global optimum. This work generalizes our monospectral restoration method [15],[16] for the multiversion images. It is seldom possible to obtain a degradation model analytically from the physics of the problem. More often a limited prior knowledge supports only some elementary assumptions about this process. Usual assumption, accepted also in this work, is that the corruption process can be modeled using a linear degradation model.

The next section introduces the image degradation model, the core part of the restoration algorithm and contains the model selection criterion. The following sections present results (3) and conclusions (4).

\section{Image Model}

Suppose $Y$ represents a true but unobservable monospectral image defined on the finite rectangular $N \times M$ underlying lattice $I$. Suppose further that we have a set of $d$ observable images $\mathcal{X}$ where each $X_{\bullet}, i \in \mathcal{X}$ is the i-th version of $Y$ distorted by the unknown PSF and noise independent of the signal. The notation - has the meaning of all possible values of the corresponding multiindex (e.g. the multiindex $r=\left\{r_{1}, r_{2}\right\}$ which has the row and columns indices, respectively). We assume knowledge of all pixels elements from the reconstructed scene. For the treatment of the more difficult problem when some data are missing see [17], [18]. The image degradation is supposed to be approximated by the linear discrete spatial domain degradation model

$$
X_{r, \bullet}=\sum_{s \in I_{r}} H_{s} Y_{r-s}+\epsilon_{r, \bullet}
$$

where $H$ is a discrete representation of the unknown point-spread function, $X_{r,}$ • is the $d \times 1$ vector of the $\mathrm{r}$-th pixel in different distortions and $Y_{r-s}$ are ideal (unobservable) image pixels. The point-spread function is assumed to be either homogeneous or it can be non-homogeneous but in this case we assume its slow changes relative to the size of an image. $I_{r}$ is some contextual support set, and a noise vector $\epsilon$ is uncorrelated with the true image, i.e., $E\left\{Y \epsilon_{\bullet}, i\right\}=0$. The point-spread function is unknown but such that we can assume the unobservable image $Y$ to be reasonably well approximated by the expectation of the corrupted image 


$$
\hat{Y}=E\left\{X_{\bullet}, i\right\}
$$

in regions with gradual pixel value changes and i-th degraded image $X_{\bullet}, i \in \mathcal{X}$ is the least degraded image from the set $\mathcal{X}$. The index $i$ of the least degraded image is excluded from the next equations (3)-(7), (10)-(19) to simplify corresponding notation. The above method (2) changes all pixels in the restored image and thus blurs discontinuities present in the scene although to much less extent than the classical restoration methods due to our restoration model (8) adaptivity. This excessive blurring can be avoided if pixels with steep step discontinuities are left unrestored, i.e.,

$$
\hat{Y}_{r}=\left\{\begin{array}{ll}
E\left\{X_{r}\right\} & \text { if }(4) \text { holds } \\
X_{r} & \text { otherwise }
\end{array},\right.
$$

where the condition (4) is

$$
p\left(X_{r} \mid X^{(r-1)}\right)>\kappa,
$$

and where $\kappa$ is a probabilistic threshold based on the prediction density. The expectation (2) can be expressed as follows:

$$
E\{X\}=\int\left(\begin{array}{cccc}
x_{1} & x_{2} & \ldots & x_{M} \\
x_{M+1} & x_{M+2} & \ldots & x_{2 M} \\
\vdots & \vdots & \ddots & \vdots \\
x_{N M-M+1} & x_{N M-M+2} & \ldots & x_{N M}
\end{array}\right) \prod_{r=1}^{N M} p\left(x_{r} \mid X^{(r-1)}\right) d x_{1} \ldots d x_{N M}
$$

where $X^{(r-1)}=\left\{X_{r-1}, \ldots, X_{1}\right\}$ is a set of noisy pixels in some chosen but fixed ordering. For single matrix elements in (5) it holds

$$
E\left\{X_{j}\right\}=\int x_{j} \prod_{r=1}^{N M} p\left(x_{r} \mid x^{(r-1)}\right) d x_{1} \ldots d x_{N M}=E_{X^{(j-1)}}\left\{E_{X_{j}}\left\{X_{j} \mid X^{(j-1)}\right\}\right\}
$$

Let us approximate after having observed $x^{(j-1)}$ the $\hat{Y}_{j}=E\left\{X_{j}\right\}$ by the conditional expectation $E\left\{X_{j} \mid X^{(j-1)}=x^{(j-1)}\right)$ where $x^{(j-1)}$ are known past realization for $j$. Thus we suppose that all other possible realization $x^{(j-1)}$ than the true past pixel values have negligible probabilities. This assumption implies conditional expectations approximately equal to unconditional ones, i.e., then the expectation (6) is $E\left\{X_{j}\right\} \approx E\left\{X_{j} \mid X^{(j-1)}\right\}$, and

$$
\hat{Y}=E\{X\} \approx\left(\begin{array}{ccc}
E\left\{X_{1} \mid x^{(0)}\right\} & \ldots & E\left\{X_{M} \mid x^{(M-1)}\right\} \\
E\left\{X_{M+1} \mid x^{(M)}\right\} & \ldots & E\left\{X_{2 M} \mid x^{(2 M-1)}\right\} \\
\vdots & \ddots & \vdots \\
E\left\{X_{N M-M+1} \mid x^{(N M-M)}\right\} & \ldots & E\left\{X_{N M} \mid x^{(N M-1)}\right\}
\end{array}\right)
$$

Suppose further that a noisy image can be represented by an adaptive $2.5 \mathrm{D}$ causal simultaneous autoregressive model 


$$
X_{r, i}=\sum_{s \in I_{r}^{c}} A_{s} X_{r-s, \bullet}+\epsilon_{r},
$$

where $\epsilon_{r}$ is a white Gaussian noise vector with zero mean, and a constant but unknown covariance matrix $\Sigma$. The noise vector is uncorrelated with data from a causal neighbourhood $I_{r}^{c}$. $A_{s}=\left[a_{s, 1}, \ldots, a_{s, d}\right] \quad \forall s$ are parameter vectors. The model adaptivity is introduced using the standard exponential forgetting factor technique in parameter learning part of the algorithm. The model can be written in the matrix form

$$
X_{r, i}=\gamma Z_{r}+\epsilon_{r}
$$

where

$$
\begin{aligned}
& \gamma=\left[A_{1}, \ldots, A_{\eta}\right], \\
& \eta=\operatorname{card}\left(I_{r}^{c}\right)
\end{aligned}
$$

is a $1 \times d \eta$ parameter matrix and $Z_{r}$ is a corresponding vector of $X_{r-s}$. To evaluate conditional mean values in (7) the one-step-ahead prediction posterior density $p\left(X_{r} \mid X^{(r-1)}\right)$ is needed. If we assume the normal-gamma parameter prior for parameters in (8) (alternatively we can assume the Jeffrey's parameter prior) this posterior density has the form of Student's probability density

$$
\begin{gathered}
p\left(X_{r} \mid X^{(r-1)}\right)=\frac{\Gamma\left(\frac{\beta(r)-d \eta+3}{2}\right) \pi^{-\frac{1}{2}} \lambda_{(r-1)}^{-\frac{1}{2}}}{\Gamma\left(\frac{\beta(r)-d \eta+2}{2}\right)\left(1+Z_{r}^{T} V_{z z(r-1)}^{-1} Z_{r}\right)^{\frac{1}{2}}} \\
\left(1+\frac{\left(X_{r}-\hat{\gamma}_{r-1} Z_{r}\right)^{T} \lambda_{(r-1)}^{-1}\left(X_{r}-\hat{\gamma}_{r-1} Z_{r}\right)}{1+Z_{r}^{T} V_{z z(r-1)}^{-1} Z_{r}}\right)^{-\frac{\beta(r)-d \eta+3}{2}},
\end{gathered}
$$

with $\beta(r)-d \eta+2$ degrees of freedom, where the following notation is used:

$$
\begin{aligned}
\beta(r) & =\beta(0)+r-1, \\
\hat{\gamma}_{r-1}^{T} & =V_{z z(r-1)}^{-1} V_{z x(r-1)}, \\
V_{r-1} & =\tilde{V}_{r-1}+I, \\
\tilde{V}_{r-1} & =\left(\begin{array}{cc}
\tilde{V}_{x x(r-1)} & \tilde{V}_{z x(r-1)}^{T} \\
\tilde{V}_{z x(r-1)} & \tilde{V}_{z z(r-1)}
\end{array}\right), \\
\tilde{V}_{u w(r-1)} & =\sum_{k=1}^{r-1} U_{k} W_{k}^{T}, \\
\lambda_{(r)} & =V_{x(r)}-V_{z x(r)}^{T} V_{z(r)}^{-1} V_{z x(r)} .
\end{aligned}
$$

where $\beta(0)>1$ and $U, W$ denote either $X$ or $Z$ vector, respectively. If $\beta(r-1)>$ $\eta$ then the conditional mean value is

$$
E\left\{X_{r} \mid X^{(r-1)}\right\}=\hat{\gamma}_{r-1} Z_{r}
$$


and it can be efficiently computed using the following recursion

$$
\hat{\gamma}_{r}^{T}=\hat{\gamma}_{r-1}^{T}+\frac{V_{z(r-1)}^{-1} Z_{r}\left(X_{r}-\hat{\gamma}_{r-1} Z_{r}\right)^{T}}{1+Z_{r}^{T} V_{z(r-1)}^{-1} Z_{r}} .
$$

The selection of an appropriate model support $\left(I_{r}^{c}\right)$ is important to obtain good restoration results. If the contextual neighbourhood is too small it can not capture all details of the random field. Inclusion of the unnecessary neighbours on the other hand adds to the computational burden and can potentially degrade the performance of the model as an additional source of noise. The optimal Bayesian decision rule for minimizing the average probability of decision error chooses the maximum posterior probability model, i.e., a model $M_{i}$ corresponding to $\max _{j}\left\{p\left(M_{j} \mid X^{(r-1)}\right)\right\}$. If we assume uniform prior for all tested support sets (models) the solution can be found analytically. The most probable model given past data is the model $M_{i}\left(I_{r, i}^{c}\right)$ for which $i=\arg \max _{j}\left\{D_{j}\right\}$.

$D_{j}=-\frac{1}{2} \ln \left|V_{z(r-1)}\right|-\frac{\alpha(r)}{2} \ln \left|\lambda_{(r-1)}\right|+\frac{d \eta}{2} \ln \pi\left[\ln \Gamma\left(\frac{\alpha(r)}{2}\right)-\ln \Gamma\left(\frac{\beta(0)-d \eta+2}{2}\right)\right]$,

where $\alpha(r)=\beta(r)-d \eta+2$.

\section{$3 \quad$ Results}

For the experiments on real data a set of the short-exposure $\left(t_{\text {exp }}=10 \mathrm{~ms}\right)$ solar images has been used. The set consist of four images (Fig.2) observed in the blue part of visible spectra $(\lambda=450.7 \mathrm{~nm})$ with resolution 0.041 " / pixel. Time sequence of the whole set is about 70s. During the standard preprocessing chain all images were corrected by MTF of the telescope, no aberration and seeing corrections have been applied. Because there is no ideal short-exposure solar image available to verify restoration results, we have chosen the most similar skin texture (Fig.1-upper left, both sets of images are presented here in different measure) from our Prague Texture Database (about 1000 prime textures) and artificially tried to simulate solar image degradations (Fig.1). The "ideal" prime skin texture was used to verify the integral restoration criterion (20) as well as the restoration quality itself.

As an objective measure of the restoration performance an integral of a sum of image partial derivatives [19] has been used:

$$
D(\hat{Y})=\iint\left(\left|\frac{\partial \hat{Y}}{\partial r_{1}}\right|+\left|\frac{\partial \hat{Y}}{\partial r_{2}}\right|\right) d r_{1} d r_{2}
$$

If the unknown point-spread function $H$ is non-negative, i.e., $H_{s} \geq 0 \forall s$ and it preserves the image energy $\left(\iint H_{r} d r_{1} d r_{2}=1\right)$ then $D(Y * H) \leq D(Y)$. This means that for the less blurred images the $D$ is growing up. Numerical (absolute mean differences between ideal and degraded images) as well as 

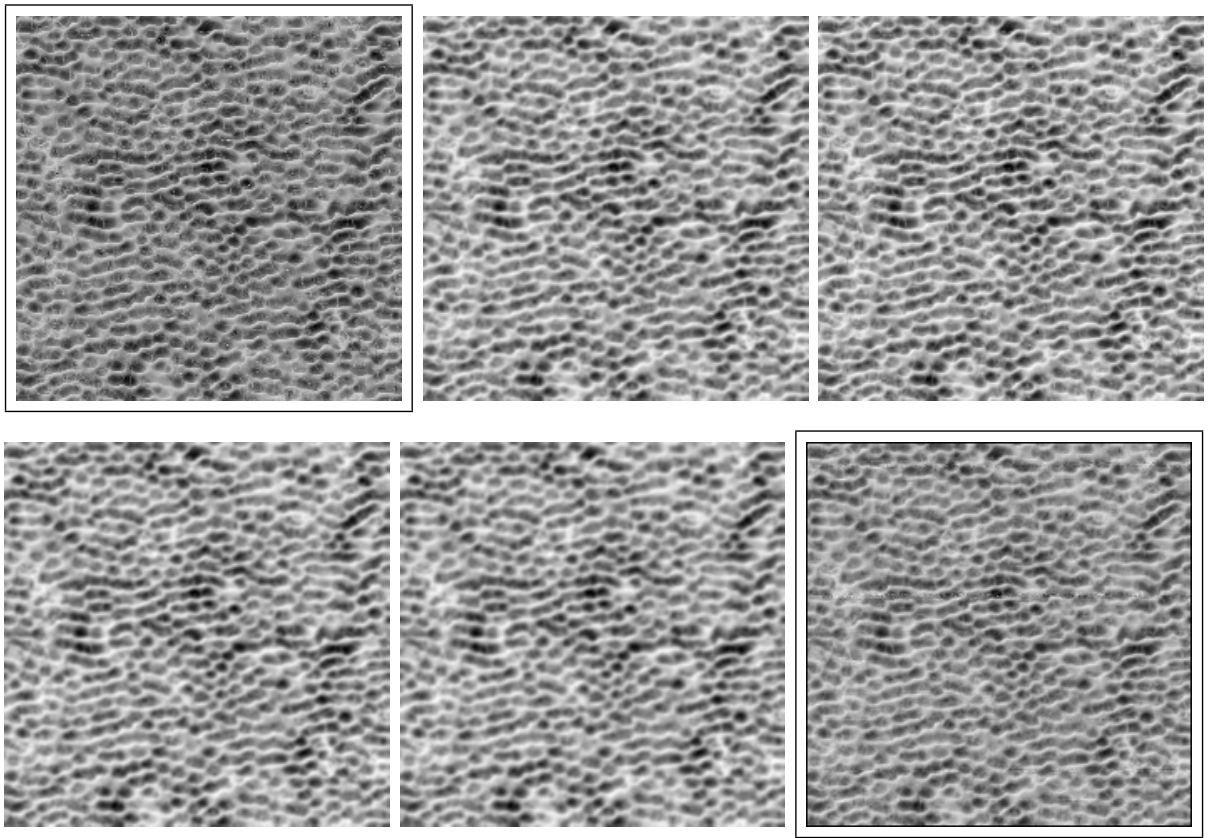

Fig. 1. Skin texture (framed upper row left) artificially degraded with Gaussian convolution filter $\left(5 \times 5, \sigma^{2}=1 ; 7 \times 7, \sigma^{2}=1\right.$ upper row, $7 \times 7, \sigma^{2}=2 ; 11 \times 11, \sigma^{2}=1$ bottom row) and the reconstructed image (framed bottom row right), respectively

the visual evaluation suggest that this criterion can serve as the crude restoration quality estimator. Tab. 1 contains its value for all presented images in the paper.

Solar images Fig.2 were reconstructed using the presented algorithm with probabilistic threshold $\kappa=0.05$ which left $54 \%$ observation pixels unchanged. The best one from the sunspot set (Fig.2-upper left) served as the reference image for the algorithm. Reconstruction result is in Fig.3 together with the corresponding criterion value in Tab.1. Fig.3-right shows gray level coded predictor probabilities which served to control the restoration switching. The lighter shades represent higher predictor probabilities while dark areas were not changed during the reconstruction. Visual comparison of the reconstructed images with the input degraded images as well as the criterion value demonstrate clear deblurring effect of the presented algorithm and restoration improvement over all used solar measurements. The proposed method was superior over the classical methods (e.g. several low pass filters, pixelwise averaging; blind deconvolution not presented here) using both criteria (20) and the visual one. The Tab. 1 demonstrates the improvement of the deblurring and noise removal effect of the presented algorithm over the real observed image data. The proposed method is clearly superior for the degraded images. 

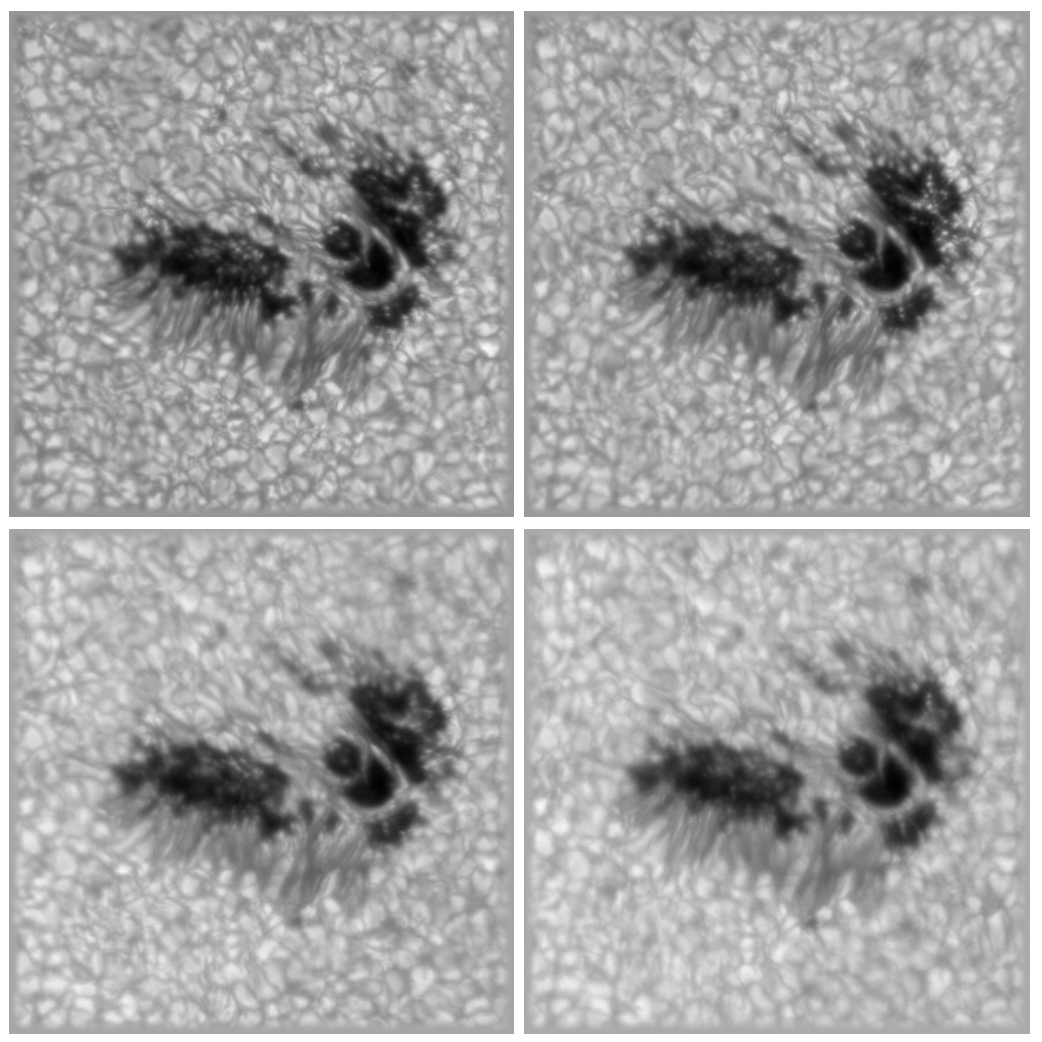

Fig. 2. The measured degraded sunspot multitemporal images (courtesy of M. Sobotka)

Table 1. The measure of the restoration quality $D$ evaluated for blurred and restored images

\begin{tabular}{|l|c|c|}
\hline \multicolumn{3}{|c|}{ Sunspot and Skin-texture image restoration } \\
Image & Restored / Unrestored & $D$ \\
\hline Fig.1 upper left (ideal) & $\mathrm{U}$ & 88.66 \\
Fig.1 upper middle & $\mathrm{U}$ & 62.82 \\
Fig.1 upper right & $\mathrm{U}$ & 70.06 \\
Fig.1 lower left & $\mathrm{U}$ & 58.56 \\
Fig.1 lower middle & $\mathrm{U}$ & 55.84 \\
Fig.1 lower right & $\mathrm{R}$ & $\mathbf{7 7 . 1 7}$ \\
\hline Fig.2 upper left & $\mathrm{U}$ & 24.24 \\
Fig.2 upper right & $\mathrm{U}$ & 23.25 \\
Fig.2 lower left & $\mathrm{U}$ & 21.39 \\
Fig.2 lower right & $\mathrm{U}$ & 20.65 \\
Fig.3 left & $\mathrm{R}$ & $\mathbf{2 5 . 6 1}$ \\
\hline
\end{tabular}



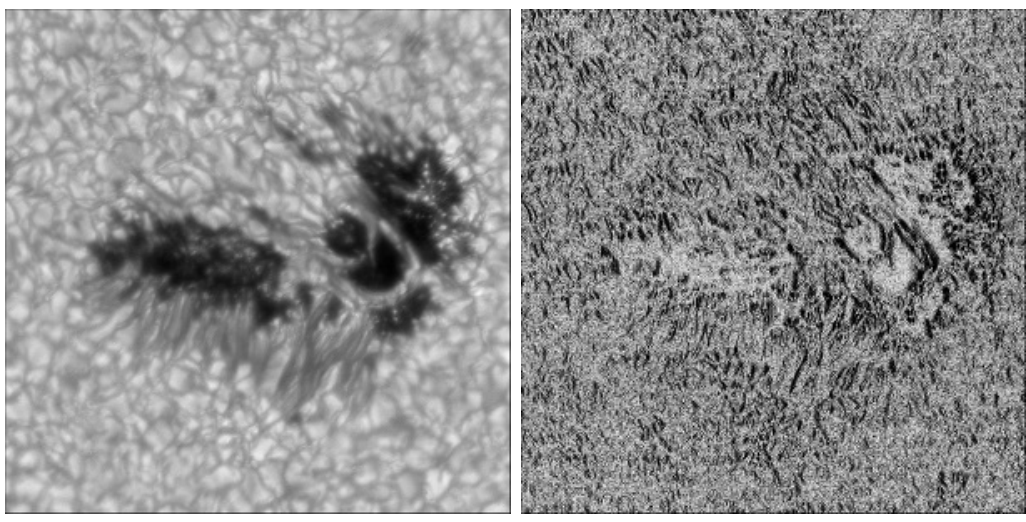

Fig. 3. The reconstructed sunspot image using our method and its corresponding prediction probability image

\section{Conclusions}

The proposed recursive multitemporal blur minimizing reconstruction method is very fast (approximately five times faster than the median filter) robust and its reconstruction results surpasses some standard reconstruction methods, which we were able to implement for verification. Causal models such as [17] have obvious advantage to have the analytical solution for parameter estimation, prediction, or model identification tasks. However, this type of models may introduce some artifacts in restored images. These undesirable effects are diminished by introducing adaptivity into the model. This novel formulation allow us to obtain extremely fast adaptive multichannel / multitemporal restoration and it can be easily parallelized. The method can be also easily and naturally generalized for multispectral (e.g. colour, multispectral satellite images) or registered images which is seldom the case for alternative methods. Finally, this method enables to estimate homogeneous or slowly changing non-homogeneous degradation point-spread function (not presented here). Although our preliminary results are very promising, comparison with sophisticated recent alternatives and testing on larger observation sequences is still needed to confirm our conclusions.

\section{Acknowledgments}

This research was supported by the EC projects no. IST-2001-34744 RealReflect, FP6-507752 MUSCLE and partially by the grants no. A2075302 of the Grant Agency of the Academy of Sciences CR, GACR no. 102/04/0155, MŠMT 1M6798555601, and AV CR project 1QS300120506. 


\section{References}

1. Fried, D. J. Opt. Soc. Am. 56 (1966)

2. Roddier, F. In Wolf, E., ed.: Progress in Optics. Volume XIX., Nort-Holland (1981)

3. Schulz, T.: Multiframe blind deconvolution of astronomical images. J. Opt. Soc. Am. A 10 (1993) 1064-1073

4. Perona, P.: Deformable kernels for early vision. IEEE Trans. Pattern Anal. Mach. Int. 17 (1995) 488-489

5. Perona, P., Malik, J.: Scale-space and edge detection using anisotropic diffusion. IEEE Trans. Pattern Anal. Mach. Int. 12 (1990) 629-639

6. Fischl, B., Schwartz, E.: Learning an integral equation approximation to nonlinear anisotropic diffusion in image processing. IEEE Trans. Pattern Anal. Mach. Int. 19 (1997) 342-352

7. Nitzberg, M., Shiota, T.: Nonlinear image filtering with edge and corner enhancement. IEEE Trans. Pattern Anal. Mach. Int. 16 (1992) 826-833

8. Andrews, H.C., Hunt, B.: Digital Image Restoration. Prentice-Hall, Englewood Cliffs (1977)

9. Hunt, B.: The application of constraint least square estimation to image restoration by digital computer. IEEE Trans. Computers 22 (1973) 805-812

10. Chellappa, R., Kashyap, R.: Digital image restoration using spatial interaction models. IEEE Trans. Acoustics, Speeech and Sig. Proc. 30 (1982) 284-295

11. Geman, S., Geman, D.: Stochastic relaxation , gibbs distributions and bayesian restoration of images. IEEE Trans. Pattern Anal. Mach. Int. 6 (1984) 721-741

12. Geman, D.: Random fields and inverse problems in imaging. Springer, Berlin (1990)

13. Jeffs, B., Pun, W.: Simple shape parameter estimation from blurred observations for a generalized gaussian mrf image prior used in map restoration. In: Proc. IEEE CVPR Conf., San Francisco, IEEE (1996) 465-468

14. Lagendijk, R., Biemond, J., Boekee, D.: Identification and restoration of noisy blurred images using the expectation-maximization algorithm. IEEE Trans. on Acoust., Speech, Signal Processing 38 (1990) 1180-1191

15. Haindl, M.: Recursive model-based image restoration. In Sanfeliu, A., Villanueva, J., Vanrell, M., Alquezar, R., Huang, T., Serra, J., eds.: Proceedings of the 15th IAPR Int. Conf. on Pattern Recognition. Volume III., Los Alamitos, IEEE Press (2000) 346-349

16. Haindl, M.: Recursive model-based colour image restoration. In Caelli, T., Amin, A., Duin, R.P.W., eds.: Structural, Syntactic, and Statistical Pattern Recognition. Proceedings, Berlin, Springer (2002) 617-626

17. Haindl, M., Šimberová, S.: A high - resolution radiospectrograph image reconstruction method. Astronomy and Astrophysics, Suppl.Ser. 115 (1996) 189-193

18. Haindl, M., Šimberová, S.: A scratch removal method. Kybernetika 34 (1998) 423-428

19. Subbarao, M., Choi, T., Nikzad, A.: Focusing techniques. J. Optical Eng. 32 (1993) 2824-2836 